\title{
A Preliminary Comparative Study of the Electron-Cloud Effect for the PSR, ISIS, and the ESS*
}

\author{
M. A. Furman† LBNL, Berkeley, CA 94720, USA \\ and \\ M. T. F. Pivi
}

June 24, 2003

\begin{abstract}
We present preliminary electron-cloud simulation results for the Proton Storage Ring (PSR) at LANL, the ISIS synchrotron at RAL, and the European Spallation Source (ESS). For each machine, we simulate the build-up and dissipation of the electron cloud (EC) in a representative field-free section of the vacuum chamber. For all three cases, we choose the same residual gas temperature, secondary emission yield (SEY), and secondary emission spectrum. Other variables such as proton loss rate, bunch profile, intensity and energy, residual gas pressure and chamber geometry, are set at the corresponding values for each machine. Under these assumptions, we conclude that, of the three machines, the PSR is the most severely affected by the electron cloud effect (ECE), followed by the ESS, with ISIS in third place. We illustrate a strong sensitivity of the ECE to the longitudinal bunch profile by choosing two different shapes for the case of the PSR, and a weak sensitivity to residual gas pressure and proton loss rate. This preliminary study does not address the ECE in other regions of the machine, nor the beam instability that might arise from the EC.
\end{abstract}

\section{Introduction.}

The existence of the ECE has been firmly established at various storage rings [1-3]. Generally speaking, this effect is the result of the coupling of the beam with a cloud of electrons in the vacuum chamber that contains it, and leads to various undesirable effects. In particular, it is now clear that the electrons play an important role in the high-intensity instability that has been observed in the PSR storage ring for more than 13 years [4]. This instability is a particular manifestation of the ECE.

At the ISIS spallation neutron source [5], instabilities due to the presence of electrons are not reported. Nevertheless, a future upgrade of the machine [6] may be affected by this limiting effect hence electron-cloud simulation studies have been initiated for this machine in order to assess its importance.

In this article we present simulation results for the PSR, ISIS and the ESS [7] obtained with the EC code POSINST $[8,9]$ that has been developed initially at LBNL, and lately in collaboration with SLAC, over the past 7 years. The simulations presented here address only the EC development and dissipation for a representative field-free region of the vacuum chamber. We do not address the EC in other regions of the machine, notably magnetic-field regions, nor do we address the potential beam instability that might arise from the EC. The approximation of simulating one section of the machine at a time is reasonable when the average electron motion in the direction of the beam can be neglected compared with the transverse motion; this approximation is well satisfied in the cases presented here. For the cases of ISIS and the ESS, we present simulation results only at the injection stage when proton losses, and hence the ECE, are expected to be strongest.

A comparative study of a similar nature, which does address the beam instability, and which encompasses several other high-intensity proton rings, has been recently undertaken by Ohmi, Toyama and Ohmori using

\footnotetext{
*Work supported by the US DOE under contracts DE-AC03-76SF00098 and DE-AC03-76SF00515.

†mafurman@lbl.gov

${ }_{\ddagger}^{\ddagger}$ mpivi@slac.stanford.edu
} 
a more simplified model of the electron dynamics, geometry, and secondary emission [10]. In this article, on the other hand, we do take into account the EC space-charge forces, the chamber geometry, and a detailed model for the secondary emission process at the walls of the chamber that includes the contributions from backscattered electrons $[11,12]$. Concerning the SEY, we assume here a peak value $\delta_{\max }=1.5$, which may correspond to well-conditioned stainless steel surface, and a corresponding value $\delta(0)=0.37$ for the SEY at zero energy. A more realistic value for $\delta_{\max }$ might be $\gtrsim 1.7$, which we intend to study in the future along with the other missing issues mentioned above.

Our confidence in the reliability of the POSINST simulation results is based on several experimental and simulation studies of the ECE for the APS $[13,14]$ and especially the PSR. In this latter case, we have compared our results against those from another code [15], against experimental data on the electron flux at the wall of the vacuum chamber [16-18] obtained by means of a retarding-field analyzer [19], and against experimental data on the electron-cloud density in the bulk of the chamber [20-23]. From such comparisons we can assess the effects of several important parameters at the walls of the chamber such as the SEY, the proton loss rate and electron yield, and we can infer details of the electron cloud in the vicinity of the proton beam, such as the local beam neutralization level. Generally speaking, for reasonable choices for various input parameters, the agreement between our simulations and measurements is within $\sim 50 \%$ or better, depending on which physical quantity one looks at. Therefore, having calibrated our simulations against the PSR experience, we are reasonably confident that a simulated comparison between the PSR and the other machines discussed here gives us valid information on the relative importance of the ECE.

\section{Physical Model.}

For the machines considered in this article, the development of the electron cloud is seeded primarily by two sources of primary electrons, namely: (1) electrons produced by residual gas ionization, and (2) electrons produced by stray protons striking the vacuum chamber at grazing angles. Although POSINST also accommodates photoelectron emission, this source is wholly negligible in the cases presented here. Furthermore, in this preliminary assessment, we neglect electrons produced in the injection region by the stripping foil, as this is a localized source hence is not expected to dominate the ECE as a whole.

Once the electron cloud gets started by any of these processes, it can get significantly amplified by the compounding effect of secondary electron emission, which occurs when electrons strike the vacuum chamber. The importance of this process depends on a combination of several variables, such as: (1) the intensity, transverse size and longitudinal profile of the bunch; (2) the fill pattern of the beam (for multibunch beams); (3) the length of the gap between two successive bunches; (4) the size and shape of the vacuum chamber cross section; and (5) the SEY and secondary electron spectrum. In particular, for the kinds of machines discussed here, which make use of long, intense bunches, the phenomenon of trailing-edge multipacting can be quite significant, as is the case for the PSR [4]. The mechanism is sketched in Fig. 1, and a simulated movie can be found in Ref. 24. This phenomenon depends sensitively on the SEY, and is a close relative of beam-induced multipacting $[25,26]$. In this article all the above-mentioned variables are taken into account by appropriate parameter choices and realistic modeling, hence we are confident that this important effect is well described by our simulations.

\subsection{Sources of Electrons.}

The contribution to the population of primary electrons from stray protons striking the chamber walls is given by

$$
n_{e(p l)}^{\prime}=\eta_{\mathrm{eff}} n_{p l}^{\prime}
$$

where $n_{p l}^{\prime}$ is the number of lost protons per beam proton per unit length of beam traversal, and $\eta_{\text {eff }}$ is the effective electron yield per proton-wall collision (" $p l$ " stands for "proton loss"). Experience at the PSR [27] suggests that $\eta_{\text {eff }}$ is in the range $100-200$; in this article we choose $\eta_{\text {eff }}=100$ for definiteness for all three machines, as listed in Table 1 . As for the proton loss rate $n_{p l}^{\prime}$, experience at the PSR indicates that a fraction $\sim 4 \times 10^{-6}$ of the beam is lost per revolution; dividing by the ring circumference this yields $n_{p l}^{\prime}=4.4 \times 10^{-8} \mathrm{p} / \mathrm{m}$. Experience at ISIS [28] indicates that significant proton losses occur only during the first $\sim 600$ revolutions. During this time, $\sim 8 \%$ of the beam is lost hence $n_{p l}^{\prime}=8.2 \times 10^{-7} \mathrm{p} / \mathrm{m}$. The ESS 


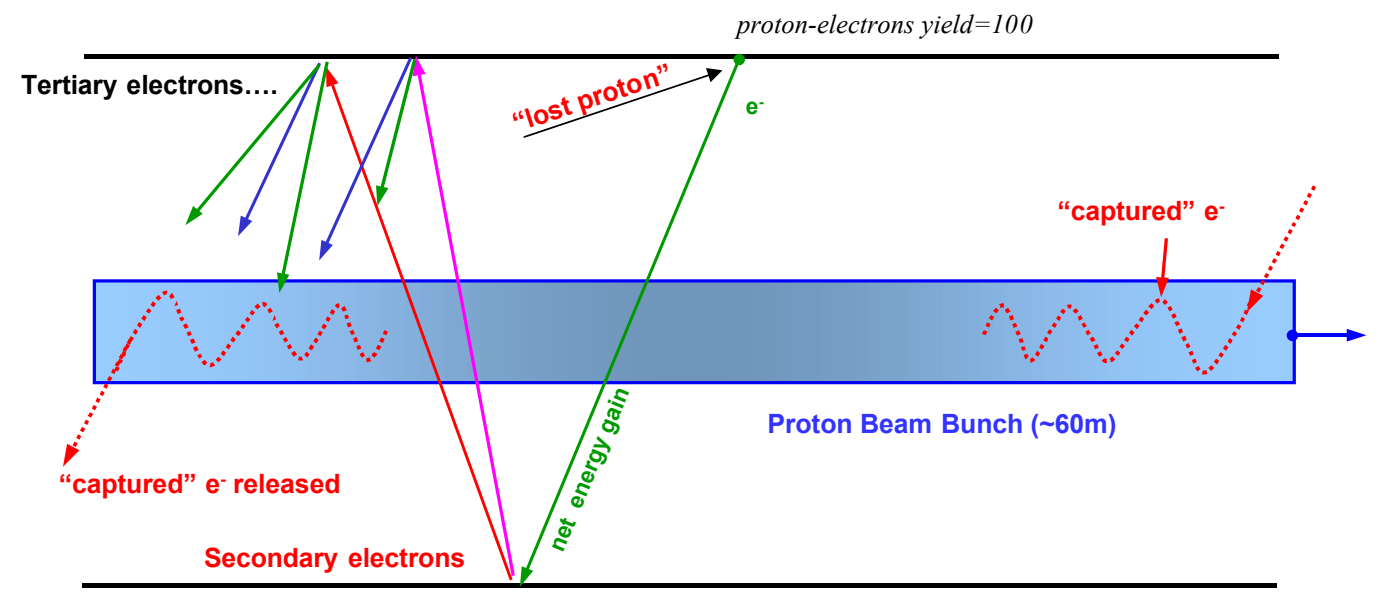

Vacuum Chamber Wall

Figure 1: Sketch of the electron multiplication mechanism in long proton bunches.

design $[28,29]$ calls for a limit of $0.01 \%$ of lost beam during the first $\sim 250$ revolutions following injection, yielding $n_{p l}^{\prime}=1.8 \times 10^{-9} \mathrm{p} / \mathrm{m}$.

The ionization probability per unit length (inverse of the mean free path) for a proton traversing a gas of density $\rho$ is given by $\rho \sigma_{i}$, where $\sigma_{i}$ is the ionization cross-section. Assuming that only one electron is generated per ionization event, and expressing $\rho$ in terms of the residual gas pressure $p$ and temperature $T$, we obtain

$$
n_{e(i)}^{\prime}\left[\mathrm{m}^{-1}\right]=3.3 \sigma_{i}[\text { Mbarn }] \times p[\text { Torr }] \times \frac{294}{T[\mathrm{~K}]}
$$

for the number of electrons generated per proton per unit length of traversal through the residual gas. We choose the typical value $\sigma_{i}=2$ Mbarns for a high-energy proton [30], and assume $T=294 \mathrm{~K}$. Pressure values are listed in Table 1.

The above-mentioned types of primary electrons are produced with different spectra, and in different parts of the chamber. These variables are properly taken into account by POSINST. Lost-proton electrons are generated at the chamber walls, and we assume that they are distributed uniformly around the chamber cross section. Ionization electrons are predominantly generated within the beam. As for the time distribution of the electron production at a given location in the ring, we assume the proportionality

$$
n_{e(p l)}^{\prime}, n_{e(i)}^{\prime} \propto \lambda_{b}(t)
$$

where $\lambda_{b}(t)$ is the beam line density at time $t$ at the ring location under study. This proportionality is fairly obvious for the ionization electrons; as for the lost-proton electrons, it is justified by noting that the the proton loss rate must be proportional to $\lambda_{b}$, and that the stray protons remain substantially comoving with the beam until they strike the chamber wall.

\subsection{Secondary Emission Process.}

When an electron strikes the beam pipe surface it can be absorbed, or it can generate secondary electrons. The SEY $\delta\left(E_{0}\right)$ and the corresponding emitted-electron energy spectrum $d \delta / d E\left(E_{0}=\right.$ incident electron energy, $E=$ emitted secondary energy) are represented, in our simulation, by a detailed probabilistic model, described elsewhere, whose parameters have been obtained from detailed fits to secondary emission measurements $[11,12]$.

The main SEY parameters are the energy $E_{\max }$ at which $\delta\left(E_{0}\right)$ is maximum, the peak value itself, $\delta_{\max }=\delta\left(E_{\max }\right)$, and the SEY at zero energy, $\delta(0)$. In this article we assume values corresponding to stainless steel and set $\delta_{\max }=1.5$, which might correspond to a well conditioned surface; the corresponding 
value for $\delta(0)$ is $\sim 3.7$. Although a value $\delta_{\max } \gtrsim 1.7$ might be more realistic, the lower value has the advantage of greater computational speed, and we consider it adequate for a preliminary comparative study. Figure 2 displays $\delta\left(E_{0}\right)$ along with its three main components, namely the elastic electron yield $\delta_{e}$, the rediffused electron yield $\delta_{r}$, and the the true-secondary electron yield $\delta_{t s}$.

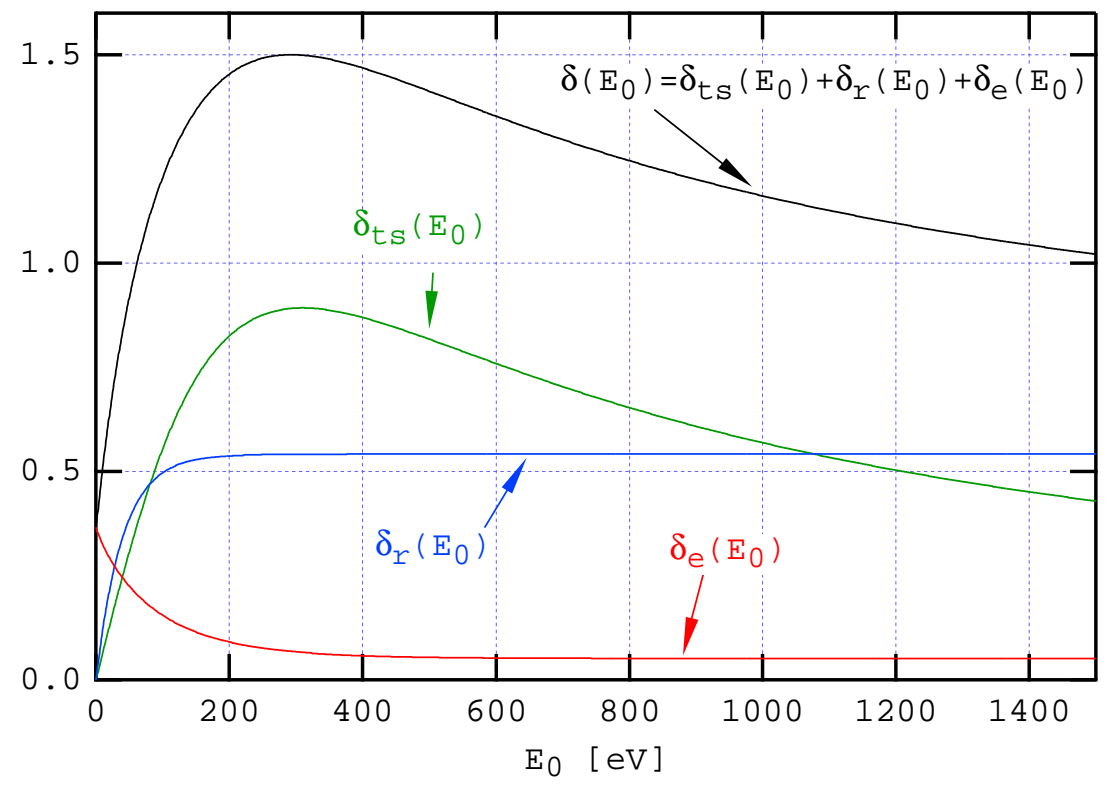

Figure 2: The SEY $\delta\left(E_{0}\right)$ and its three components used in the simulations. These were obtained from a fit to at stainless steel sample of a SLAC standard 304 rolled sheet, chemically etched and passivated but not conditioned, then scaled so that $\delta_{\max }=1.5$ (corresponding to $\delta(0)=0.37$ ). The peak occurs at $E_{0}=E_{\max }=292.6 \mathrm{eV}$.

\subsection{Simulation Model.}

In POSINST, the beam is represented by a prescribed function of space and time, while the electrons are fully dynamical, and are represented by macroparticles. The code simulates the electron cloud in a specific region of the chamber; in this article we only look at a field-free region whose length we choose to be $L=1$ m.

The beam energies we assume for ISIS and the ESS, displayed in Table 1, correspond roughly to the stage when significant proton losses are observed or expected $[28,29]$. For the PSR we use the actually measured bunch profile, while for ISIS and the ESS we simply assume a parabolic shape, as shown in Fig. 3. Table 1 shows the corresponding values for the full bunch length $\tau_{b}$, and the interbunch gap $\tau_{g}$. As for the transverse bunch profile, we use the gaussian shape with RMS values listed in Table 1. In the simulation, the bunch is divided up into $N_{k}$ kicks (i.e., $N_{k}-1$ slices), and the interbunch gap into $N_{g}$ steps. The values shown in Table 1 represent a compromise between computational accuracy and speed. For the PSR, a spot-check with $N_{k}=5001$ leads to differences $\sim 10-20 \%$ from the case $N_{k}=1001$. The values for $N_{g}$ are chosen so that the integration time step in the bunch and in the gap are approximately the same, i.e., $\Delta t=\tau_{b} /\left(N_{k}-1\right) \approx \tau_{g} / N_{g}$. The image and space-charge forces are computed and applied after each kick in the bunch and each step in the gap.

The PSR vacuum chamber is cylindrical of $5 \mathrm{~cm}$ radius. For ISIS and the ESS the chamber in the field-free regions is rectangular with an aspect ratio that follows that of the beam. Table 1 shows our chosen values for this preliminary study. Our code computes the image forces from the beam and from the electrons assuming a perfectly-conducting pipe surface of the specified cross-sectional shape.

The number of primary electrons generated during one bunch passage in a chamber section of length $L$ 
Table 1: Assumed parameters for the PSR, ISIS and ESS simulations.

\begin{tabular}{|c|c|c|c|c|}
\hline Parameter & Symbol (unit) & PSR & ISIS & ESS \\
\hline \multicolumn{5}{|l|}{ Ring and beam parameters } \\
\hline Ring circumference & $C(\mathrm{~m})$ & 90 & 163.4 & 220 \\
\hline No. bunches/beam & $\cdots$ & 1 & 2 & 1 \\
\hline Beam energy & $E(\mathrm{GeV})$ & 1.735 & 1.018 & 2.272 \\
\hline Bunch population & $N_{b}\left(10^{13}\right)$ & 5 & 1.25 & 23.4 \\
\hline Full bunch length & $\tau_{b}(\mathrm{~ns})$ & 254 & 232 & 560 \\
\hline Inter-bunch gap & $\tau_{g}(\mathrm{~ns})$ & 103 & 470 & 246 \\
\hline Longit. bunch profile & $\ldots$ & actual & parabolic & parabolic \\
\hline Beam pipe cross section & $\cdots$ & cylindrical & rectangular & rectangular \\
\hline Beam pipe semi-axes & $(a, b)(\mathrm{cm})$ & $(5,5)$ & $(6.3,8)$ & $(8.2,8.2)$ \\
\hline Transverse bunch profile & $\cdots$ & gaussian & gaussian & gaussian \\
\hline Transverse RMS bunch sizes & $\left(\sigma_{x}, \sigma_{y}\right)(\mathrm{cm})$ & $(1,1)$ & $(2.3,3.4)$ & $(2,2)$ \\
\hline Proton loss rate & $n_{p l}^{\prime}(\mathrm{p} / \mathrm{m})$ & $4.4 \times 10^{-8}$ & $8.2 \times 10^{-7}$ & $1.8 \times 10^{-9}$ \\
\hline Proton-electron yield & $\eta_{\text {eff }}$ & 100 & 100 & 100 \\
\hline Proton-loss $\mathrm{e}^{-}$creation rate & $n_{e(p l)}^{\prime}(\mathrm{e} / \mathrm{p} / \mathrm{m})$ & $4.4 \times 10^{-6}$ & $8.2 \times 10^{-5}$ & $1.8 \times 10^{-7}$ \\
\hline Residual gas pressure & $p$ (nTorr) & 10 & 500 & 75 \\
\hline Temperature & $T(\mathrm{~K})$ & 294 & 294 & 294 \\
\hline Ionization cross-section & $\sigma_{i}$ (Mbarns) & 2 & 2 & 2 \\
\hline Ionization $\mathrm{e}^{-}$creation rate & $n_{e(i)}^{\prime}(\mathrm{e} / \mathrm{p} / \mathrm{m})$ & $6.6 \times 10^{-8}$ & $3.3 \times 10^{-5}$ & $5 \times 10^{-7}$ \\
\hline Peak SEY & $\delta_{\max } \equiv \delta\left(E_{\max }\right)$ & 1.5 & 1.5 & 1.5 \\
\hline Energy at peak SEY & $E_{\max }(\mathrm{eV})$ & 292.6 & 292.6 & 292.6 \\
\hline SEY at 0 energy & $\delta(0)$ & 0.366 & 0.366 & 0.366 \\
\hline Backscattered component at $E_{\max }$ & $\delta_{e}\left(E_{\max }\right)+\delta_{r}\left(E_{\max }\right)$ & 0.61 & 0.61 & 0.61 \\
\hline \multicolumn{5}{|l|}{ Simulation parameters } \\
\hline No. kicks/bunch & $N_{k}$ & 1001 & 1001 & 2001 \\
\hline No. steps during gap & $N_{g}$ & 406 & 2026 & 879 \\
\hline No. prim. macroelectrons/bunch & $K$ & 1000 & 1000 & 1000 \\
\hline Macroelectron charge & $Q / e\left(10^{5}\right)$ & 2.26 & 55.7 & 1.58 \\
\hline Time step size & $\Delta t(\mathrm{~ns})$ & 0.25 & 0.23 & 0.28 \\
\hline
\end{tabular}

is given by

$$
N_{e}=N_{b} L n_{e}^{\prime}
$$

where $N_{b}$ is the number of protons per bunch, and $n_{e}^{\prime}=n_{e(i)}^{\prime}+n_{e(p l)}^{\prime}$ is the total number of primary electrons generated per proton per unit length of beam traversal. In our simulation, these $N_{e}$ electrons are represented by $K$ macroparticles, hence the macroparticle charge $Q$ in units of $e$ (or mass $M$ in units of the electron mass $\left.m_{e}\right)$ is given by

$$
Q / e=M / m_{e}=\frac{N_{e}}{K}=\frac{N_{b} L n_{e}^{\prime}}{K}
$$

The value for $K$ is an input to the simulation; the most faithful simulation case corresponds to $Q / e=1$, but this typically implies an excessively large value for $K$. In the cases presented here, we have chosen $K=1000$ for all three cases, which represents a reasonable compromise between computational accuracy and speed for a preliminary assessment. The corresponding values for $Q / e$ for each machine are shown in Table 1. A characteristic of POSINST is that $Q / e$ is the same for all macroelectrons, whether they are primaries or secondaries. 


\section{Results and Discussion.}

For our present purposes, we have chosen to run the simulation for only three bunch passages followed by a time gap equivalent to one bunch passage. Most results show that the length of this simulation run is quite sufficient, for the parameters chosen, for the EC to reach an approximate steady state.

Figure 3 shows the single-bunch current profile assumed in the simulations for each case. Figure 4 shows the time-averaged electron density during the run, viewed head-on. The corresponding line density is shown in Fig. 5. The EC line density $\lambda_{e}$ rises quickly during the passage of the tail of the bunch owing to trailingedge multipacting, and dissipates during the passage of the gap. It reaches a peak value $\lambda_{e} \sim 8 \mathrm{nC} / \mathrm{m}$ for the PSR, $\lambda_{e} \sim 1 \mathrm{nC} / \mathrm{m}$ for ISIS, and $\lambda_{e} \sim 2 \mathrm{nC} / \mathrm{m}$ for the ESS. This peak value is quite sensitive to the value of $\delta_{\max }$ : a spot check for the PSR with $\delta_{\max }=1.7$ shows peak values of $\lambda_{e}$ almost an order of magnitude larger than for $\delta_{\max }=1.5$. During the passage of the bulk of the bunch, $\lambda_{e}$ is not directly affected by secondary electron emission because the electrons are effectively trapped by the beam potential hence cannot reach the vacuum chamber walls, hence $\lambda_{e}$ grows only due to the production of primary electrons. This is clearly visible for the case of ISIS in Fig. 5, which has the largest value for $n_{e}^{\prime}$ of the three machines, as seen in Table 1. In addition, ISIS has the longest interbunch gap, hence the EC dissipates by more than an order of magnitude in between bunches.

A better indicator of the beam stability is the local neutralization factor $\chi$, i.e., the number of electrons divided by the number of protons within the one-sigma ellipse about the bunch center. This is shown in Fig. 6; it is clear that the PSR has a minimum value $\chi \sim 1 \%$, while ISIS and the ESS have minimum values $\chi \sim 0.1 \%$ and $\chi \sim 0.25 \%$, respectively. One may sensibly conclude from this that ISIS and the ESS are significantly below threshold for a PSR-style instability; however, since our simulations do not address the instability, we cannot draw this conclusion with certainty.

Figure 7 shows the flux of electrons $J$ (dimensions of current/(unit area)) striking the vacuum chamber wall. One observes again that the electron flux for the PSR is much larger than for the other two machines. Measurements at the PSR show a peak value $J \sim 400 \mu \mathrm{A} / \mathrm{cm}^{2}[16,17]$, which is roughly consistent with the value shown in Fig. 7. However, the measurements are location dependent, and probably correspond to a value of $\delta_{\max }$ somewhat larger than 1.5.

Figure 8 shows the average electron-wall collision energy. In this case there is not much difference among the three cases, except that the PSR exhibits a longer time interval during which the energy is rather high. This is a direct result of the peculiar shape of the longitudinal bunch profile, as discussed below.

Finally, Fig. 9 shows the energy spectrum of the electrons striking the vacuum chamber wall, averaged over time for the entire run. The typical electron-wall collision energy is $E_{0} \sim 5 \mathrm{eV}$, which is probably dominated by the large number of electrons in the interbunch gap. The secondary peak at $E_{0} \sim 240 \mathrm{eV}$ in the case of the PSR is clearly supported by measurements, and we believe it is at least in part a consequence of the cylindrical symmetry.

\subsection{Sensitivity to the bunch profile.}

Recently we carried out a numerical investigation of the effect of the bunch profile on trailing-edge multipacting at the PSR by simulating the ECE with bunches with truncated tails keeping the bunch population constant [31]. The idea that truncating the tail breaks the resonance condition that causes multipacting was clearly supported by the simulations. Motivated by this, we have carried out one more test in this article in which we assumed that the PSR bunch profile is parabolic such that the full length $\tau_{b}$ and population $N_{b}$ are the same as the actual bunch, as shown at the top of Fig. 10. The rest of Fig. 10 plus Fig. 11 show the comparison of the various quantities discussed above for the two cases. It is evident from all the results presented that the ECE for a parabolic bunch profile is much less severe than for the actual profile, simply because a parabolic profile has a weaker tail than the actual, approximately triangular, shape. More specifically, when comparing the PSR with a parabolic bunch profile with the actual profile, the peak line density $\lambda_{e}$ is a factor $\sim 6$ lower; the line density in the bulk of the bunch is a factor $\sim 2$ lower; the local neutralization factor $\chi$ is a factor $\lesssim 2$ lower; and the electron flux at the wall $J$ is a factor $\sim 10$ lower. These results corroborate our earlier results for truncated bunches.

Furthermore, the plots of the EC line density $\lambda_{e}$ and the beam neutralization factor $\chi$ for the PSR with a parabolic bunch profile are strikingly similar to those for the ESS. In addition, noting that the electron-wall 
flux $J$ scales as $J \sim d^{-2}$, where $d$ is the characteristic transverse dimension of the chamber, one expects $J$ to be a factor $\sim 3$ lower for the ESS than for the PSR purely on geometrical considerations, which is indeed what is observed when comparing Fig. 7 (bottom) with Fig. 11 (top). When this is taken into account, one also concludes that $J$ for the ESS would be very close to $J$ for the PSR with a parabolic bunch profile if the transverse chamber dimensions were the same.

\subsection{Sensitivity to proton-loss rate.}

We carried out a spot check for ISIS in which $n_{p l}^{\prime}$ was twice the value listed in Table 1 , namely $16.4 \times 10^{-7}$ $\mathrm{p} / \mathrm{m}$, corresponding to $n_{e(p l)}^{\prime}=16.4 \times 10^{-5} \mathrm{e} / \mathrm{p} / \mathrm{m}$, while all other quantities were kept fixed at their values listed in the Table. The peak value of $\lambda_{e}$ roughly doubled as did the electron-wall flux $J$. However, the neutralization factor $\chi$ increased by only $\sim 15-20 \%$. To the extent that $\chi$ determines the instability threshold, these results are consistent with PSR experience, in which a deliberate increase in the proton loss rate did not lead to a significant concomitant change in the threshold [4].

\subsection{Sensitivity to residual gas pressure.}

We carried out a spot check in which we set the vacuum pressure for ISIS and the ESS to the same value as for the PSR, namely 10 nTorr instead of the values listed in Table 1. For the case of ISIS, in which this represents a factor 50 decrease in pressure, the results for $\lambda_{e}$ and $J$ were almost unchanged, while $\chi$ decreased by a factor $\sim 2$. For the ESS, in which this represents a decrease of a factor 7.5 in pressure, $\lambda_{e}$ was almost unchanged while $J$ decreased by a factor $\sim 2$ while $\chi$ decreased by only $\sim 15 \%$. These results show that, in the range of parameters considered here, the ECE is dominated by other mechanisms.

\section{Conclusions.}

We have presented a comparative simulation study of the ECE for the PSR, ISIS and the ESS. This study is of limited scope, as it deals with the ECE only in a field-free region of the machine, and does not attempt to address the beam instability that might arise from the EC. For all three machines we assumed the same residual gas temperature, proton-electron yield, and SEY parameters. Other parameters such as chamber geometry and size, proton loss rate, residual gas pressure, beam energy, bunch population, bunch length, etc., were set at the appropriate value for each case. For the cases of ISIS and the ESS, we have carried out the simulation only at the injection stage when proton losses, and hence the ECE, are expected to be strongest. We have only assessed the sensitivity of our results to variations in vacuum pressure, proton loss rate, and longitudinal bunch profile. Since our simulation results have previously been reasonably well calibrated against PSR experience, we can draw several useful conclusions (subject to the above caveats), namely:

1. The ECE is strongest in the PSR, followed by the ESS, with ISIS in third place.

2. It does not seem surprising that the ECE is not observed in ISIS; even if electron detectors were to be installed at the vacuum chamber walls, the expected electron signal would be a factor $\sim 50$ below that for the PSR. The weakness of the ECE in ISIS is almost certainly due to the relatively short, well separated bunches.

3. The ESS might not be subject to a PSR-style EC instability, although this is a weak conclusion.

4. If the bunch in the PSR had a parabolic time profile rather than its actual, approximately triangular, profile, the ECE would be much weaker, and the instability threshold might be significantly higher. In this case, the ECE at the PSR and the ESS would coincidentally be remarkably similar in strength.

5. By the same token, if the bunch profile in ISIS and the ESS were not parabolic (as we have assumed here) but had linear or exponential tails instead, the ECE would probably be much stronger as a consequence of enhanced trailing-edge multipacting. In this case, the ESS might well be affected by a PSR-style instability. 
6. Within a range of values, vacuum pressure and proton loss rate do not affect the beam neutralization factor very much, although they do affect the average EC density and electron-wall flux. This may imply that the instability threshold has a more subtle dependence on primary electron generation mechanisms, a fact which appears to be consistent with PSR experience.

Our conclusion that the ECE is strong at the PSR and weak at ISIS agrees with that in Ref. 10. Similarly, our results for the minimum neutralization level $\chi$ for the PSR and ISIS are approximately the same as in this publication, although we are not certain that we are using the same definition for $\chi$. On the other hand, there are qualitative differences, particularly in the time evolution of the EC. Although the discrepancies can be partially attributed to differences in the physical models used, it is clear that further work is required to reach a better quantitative understanding. In Ref. 10 the bunch profile was represented by a half-sine wave function, which has linear "tails." Given the strong sensitivity that the ECE shows to the longitudinal bunch profile for rings with very long bunches, this is another example of topics that demand further theoretical, numerical and experimental studies.

\section{Acknowledgments.}

We are indebted to G. Bellodi and C. Prior for many communications and discussions on several aspects relevant to ISIS and the ESS, and to R. Macek for continued discussions on the PSR instability. We are grateful to NERSC for supercomputer support.

\section{References}

[1] Proc. ICFA Workshop on Two-Stream Instabilities in Particle Accelerators and Storage Rings, Santa Fe, NM, Feb. 16-18, 2000; http://www.aps.anl.gov/conferences/icfa/two-stream.html

[2] Proc. Intl Workshop on Two-Stream Instabilities in Particle Accelerators and Storage Rings, KEK, Tsukuba, Japan, Sept 11-14, 2001; http://conference.kek.jp/two-stream/

[3] Proc. Mini-Workshop on Electron-Cloud Simulations for Proton and Positron Beams ECLOUD'02, CERN, Apr. 15-18, CERN Yellow Report CERN-2002-001, F. Zimmermann and G. Rumolo, eds., 2002; http://slap.cern.ch/collective/ecloud02/

[4] For summaries and further references, see the contributions by R. Macek in Refs. 1, 2 and 3.

[5] http://www.isis.rl.ac.uk/

[6] C. R. Prior et. al., "ISIS Megawatt Upgrade Plans," Proc. PAC03, Portland, OR, May 12-16, 2003, paper TPAG043.

[7] http://www.ess-europe.de/en/index.php

[8] M. A. Furman and G. R. Lambertson, "The Electron Cloud Effect in the Arcs of the PEP-II Positron Ring," LBNL-41123/CBP Note-246/PEP-II AP Note AP 97.27, November 25, 1997; KEK Proceedings 97-17, p. 170, December 1997 (Proc. MBI-97 workshop, KEK, Tsukuba, Japan, 15-18 July 1997; Y. H. Chin, ed.)

[9] M. A. Furman, "The Electron-Cloud Effect in the Arcs of the LHC," LBNL-41482/CBP Note 247/CERN LHC Project Report No. 180, May 20, 1998.

[10] K. Ohmi, T. Toyama and C. Ohmori, "Electron Cloud Instability in High Intensity Proton Rings," PRST-AB 6, 114402 (2002); erratum: 6, 029901 (2003).

[11] M. A. Furman and M. T. F. Pivi, "Probabilistic Model for the Simulation of Secondary Electron Emission," LBNL-49771/CBP Note-415, November 6, 2002; published PRST-AB v5/i12/e124404 (2002). 
[12] M. A. Furman and M. T. F. Pivi, "Simulation of Secondary Electron Emission Based on a Phenomenological Probabilistic Model," LBNL-52807/SLAC-PUB-9912, June 2, 2003, http://mafurman.lbl.gov/LBNL-52807.pdf.

[13] M. A. Furman, M. Pivi, K. C. Harkay and R. A. Rosenberg, "Electron-Cloud Measurements and Simulations for the APS", LBNL-48383, CBP Note-399, June 26, 2001. Proc. PAC01, Chicago, June 18-22, 2001, 679 .

[14] K. C. Harkay and R. A. Rosenberg, "Properties of the electron cloud in a high-energy positron and electron storage ring," PRST-AB, 6, 034402 (2003).

[15] M. Blaskiewicz, M. A. Furman, M. Pivi and R. J. Macek, "Electron-Cloud Instabilities in the Proton Storage Ring and Spallation Neutron Source," PRST-AB 6, 014203 (2003).

[16] M. A. Furman and M. T. F. Pivi, "Electron Cloud Development in the Proton Storage Ring and in the Spallation Neutron Source," LBNL-51618, October 16, 2002; PRST-AB 6034201 (2003).

[17] M. Pivi and M. A. Furman, "Electron-Cloud Simulation Results for the PSR and SNS," LBNL-51038, CBP Note-441, June 19, 2002, Ref. 3.

[18] M. Pivi and M. A. Furman, "Electron-Cloud Updated Simulation Results for the PSR, and Recent Results for the SNS," LBNL-50513, CBP Note-428, June 19, 2002; Proc. EPAC02, Paris, June 3-7, 2002, paper WEPDO006.

[19] R. A. Rosenberg, K. C. Harkay, "A rudimentary electron energy analyzer for accelerator diagnostics," Nuclear Instruments and Methods in Physics Research A 453, 507-513 (2000); http://www.elsevier.nl/gej-ng/10/33/34/92/27/26/article.pdf

[20] R. Macek, M. Borden, A. Browman, D. Fitzgerald, T.-S. Wang, T. Zaugg, K. Harkay, R. Rosenberg, "Electron Cloud Diagnostics in Use at the Los Alamos PSR," Proc. PAC03, Portland, OR, May 12-16, 2003, paper ROAB003.

[21] R. Macek, M. Borden, A. Browman, D. Fitzgerald, R. McCarady, T. Spickermann, T. Zaugg, "Status of the Experimental Studies of the Electron Cloud at the Los Alamos Proton Storage Ring," Proc. PAC03, Portland, OR, May 12-16, 2003, paper RPPB035.

[22] M. A. Furman, "Formation and Dissipation of the Electron Cloud," LBNL-51829, May 20, 2003; Proc. PAC03, Portland, OR, May 12-16, 2003, paper TOPC001.

[23] R. Macek, A. Browman, M. A. Furman, and M. T. F. Pivi, to be published.

[24] Simulated movie of the electron cloud in the PSR by M. Pivi, http://slap.cern.ch/collective/ecloud02/newschedule.html

[25] O. Gröbner, "Bunch-Induced Multipactoring," Proc. 10th Intl. Accel. Conf., Serpukhov, 1977, pp. 277282.

[26] O. Gröbner, "Bunch-Induced Multipacting," Proc. PAC97, p. 3589, Vancouver, BC, May 12-16, 1997.

[27] R. Macek, private communication.

[28] G. Bellodi, private communication.

[29] C. Prior, private communication.

[30] M. Reiser, Theory and Design of Charged Particle Beams, J. Wiley \& Sons, 1994, Sec. 4.6.

[31] M. Pivi and M. A. Furman, "Mitigation of the Electron-Cloud Effect in the PSR and SNS Proton Storage Rings by Tailoring the Bunch Profile," LBNL-52709, May 2003; presented at the PAC03, Portland, OR, May 12-16, 2003, paper RPPG024. 


\section{Disclaimer}

This document was prepared as an account of work sponsored by the United States Government. While this document is believed to contain correct information, neither the United States Government nor any agency thereof, nor The Regents of the University of California, nor any of their employees, makes any warranty, express or implied, or assumes any legal responsibility for the accuracy, completeness, or usefulness of any information, apparatus, product, or process disclosed, or represents that its use would not infringe privately owned rights. Reference herein to any specific commercial product, process, or service by its trade name, trademark, manufacturer, or otherwise, does not necessarily constitute or imply its endorsement, recommendation, or favoring by the United States Government or any agency thereof, or The Regents of the University of California. The views and opinions of authors expressed herein do not necessarily state or reflect those of the United States Government or any agency thereof, or The Regents of the University of California.

Ernest Orlando Lawrence Berkeley National Laboratory is an equal opportunity employer. 

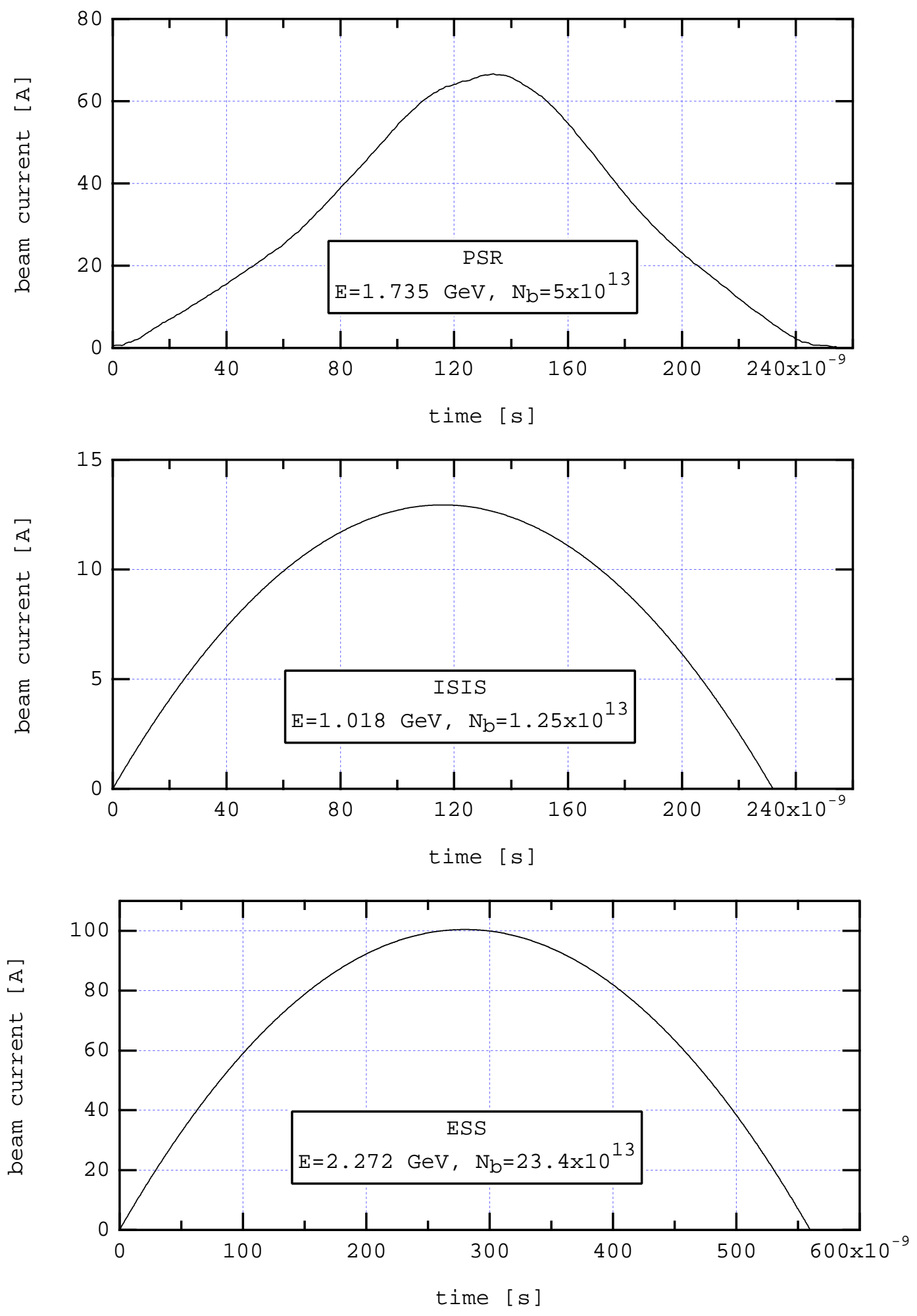

Figure 3: Single-bunch current profile assumed as input to the simulations. The PSR current was obtained from one particular set of actual measurements (data courtesy R. Macek). For ISIS and the ESS we simply assumed a parabolic shape. 

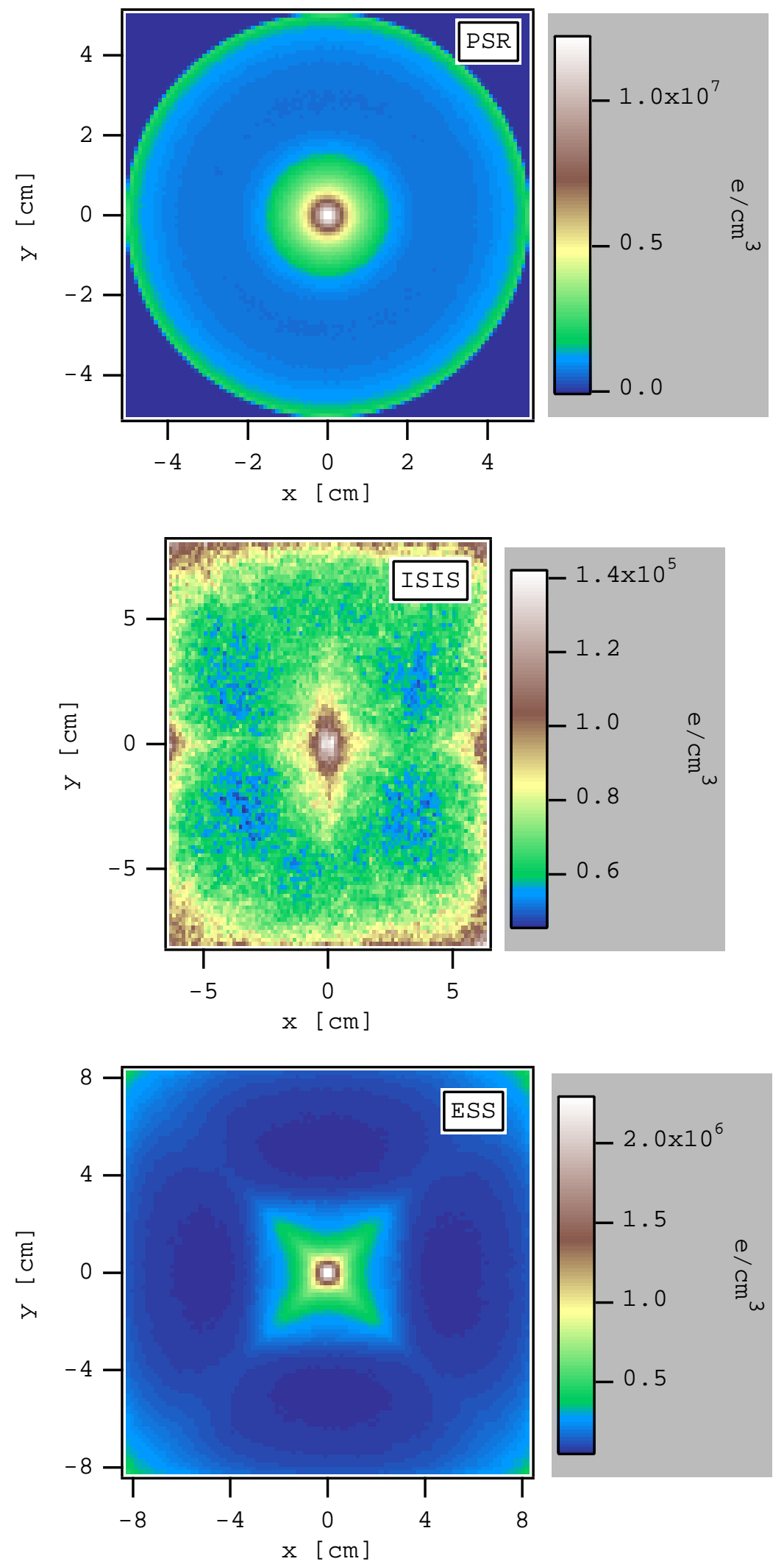

Figure 4: Time-averaged electron-cloud density. The beam is at the center of the chamber, and travels perpendicularly to the page. 

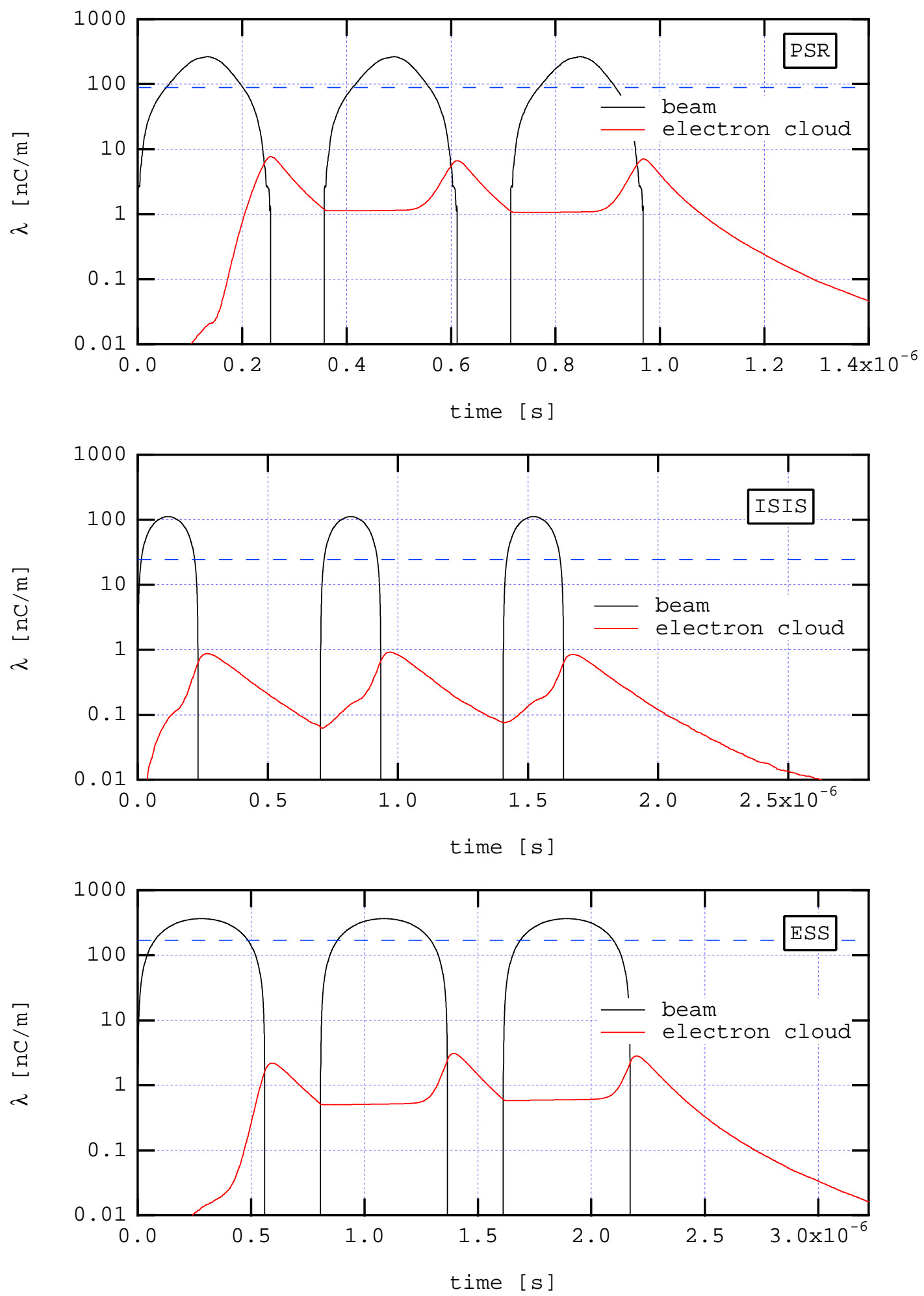

Figure 5: Beam and electron-cloud line density. A train of three bunches is injected into an empty machine, then extracted. The dashed blue line is the average beam neutrality level. 

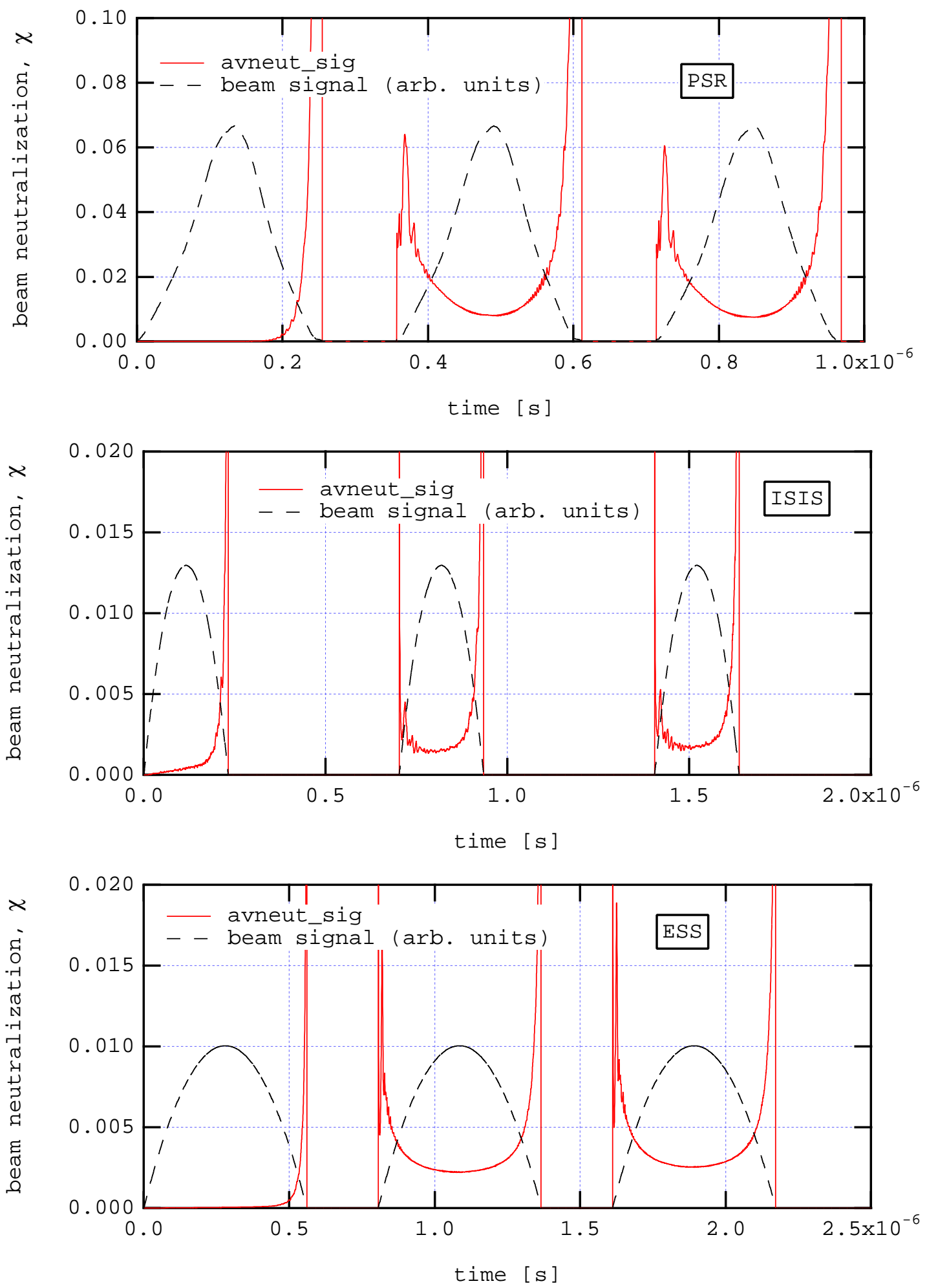

Figure 6: Beam neutralization factor, $\chi=$ (no. of electrons)/(no. of protons). Only those electrons and protons within the one- $\sigma$ ellipse of the beam center are counted. Note the factor of 5 in the vertical scale for the case of the PSR relative to the other two. The high spikes at the beginning and end of the bunch are caused by the vanishing bunch current. 

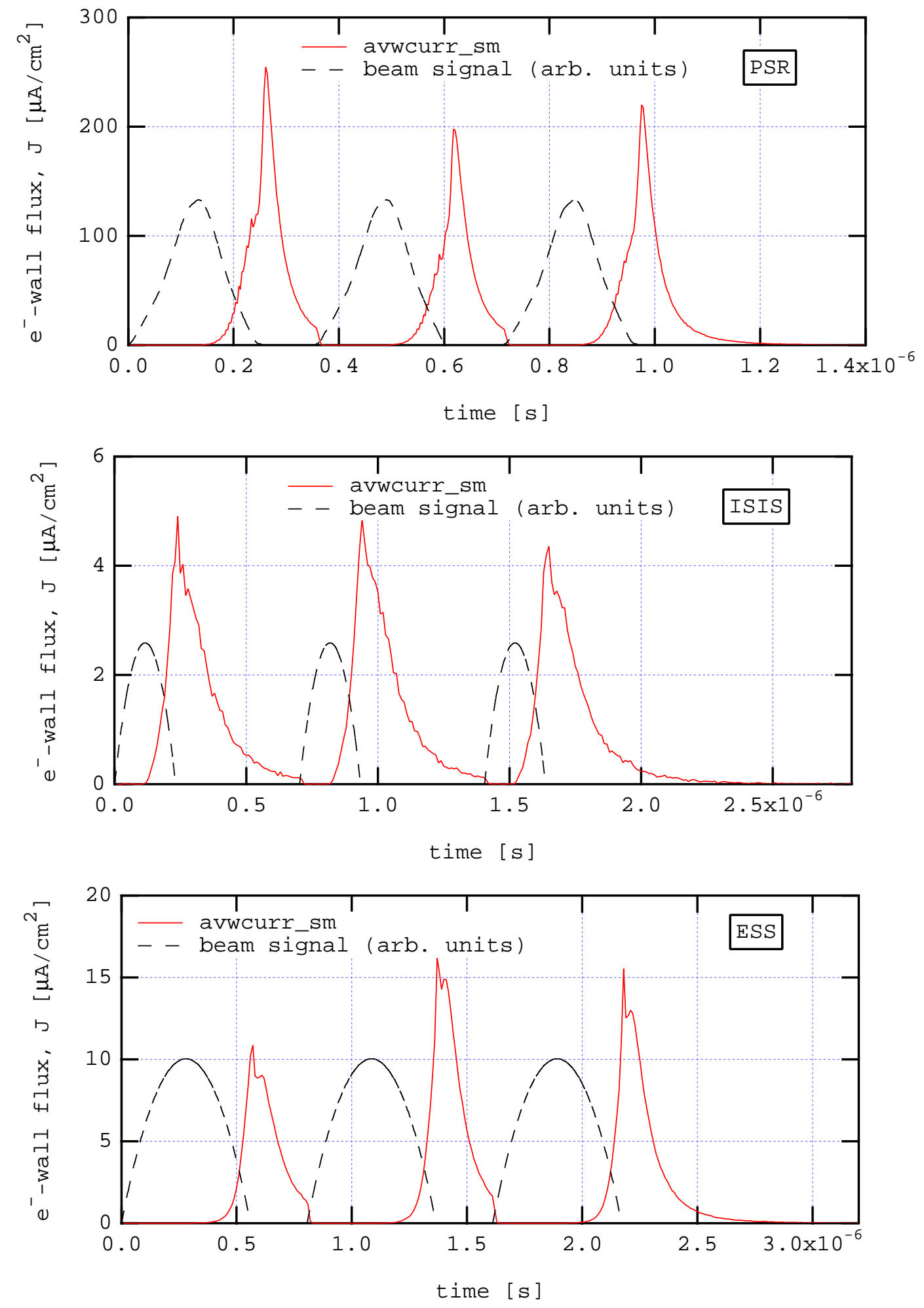

Figure 7: Average electron flux $J$ at the chamber wall. Note that the vertical scale for the PSR is much larger than for the other two. 

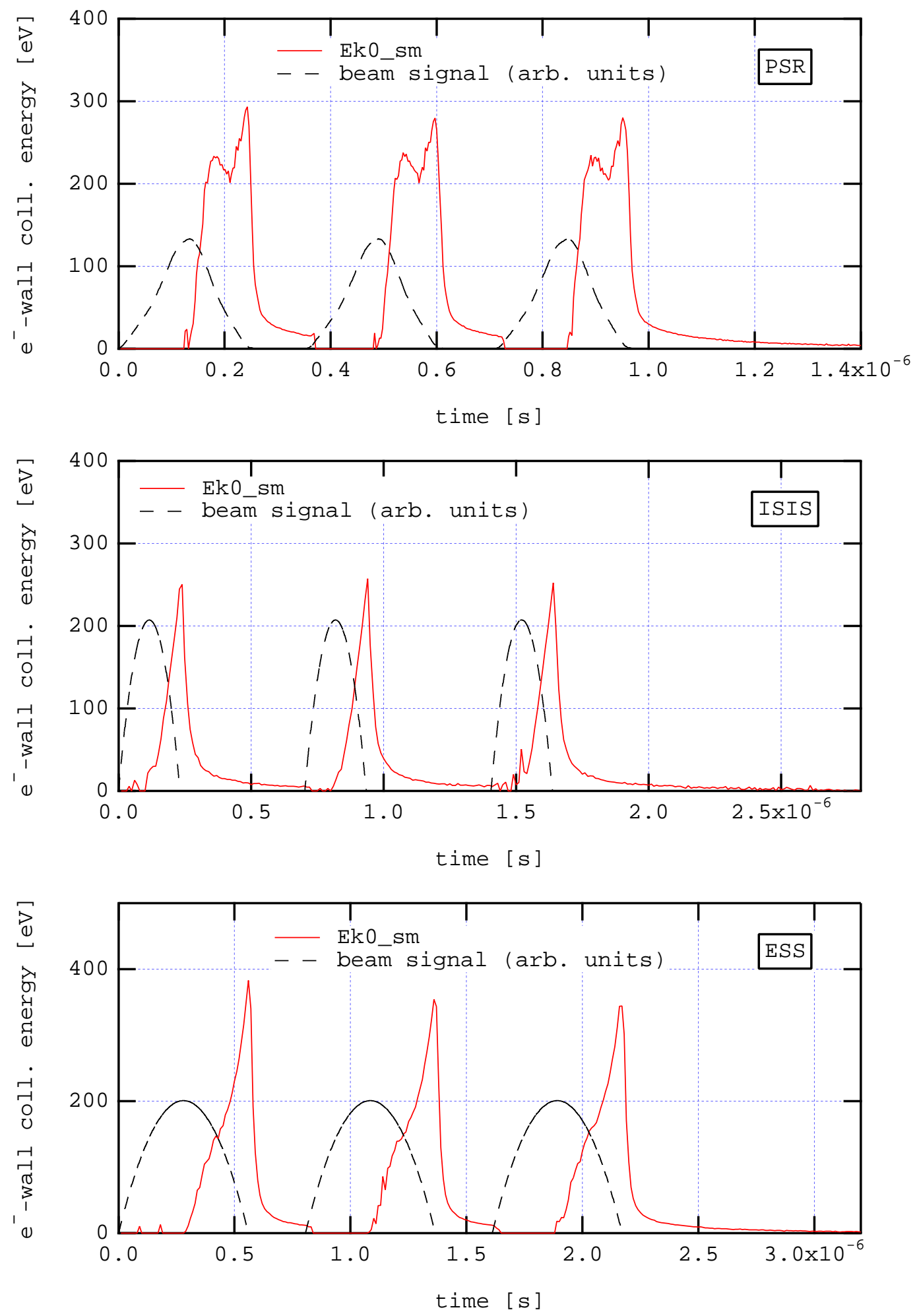

Figure 8: Average electron-wall collision energy. 

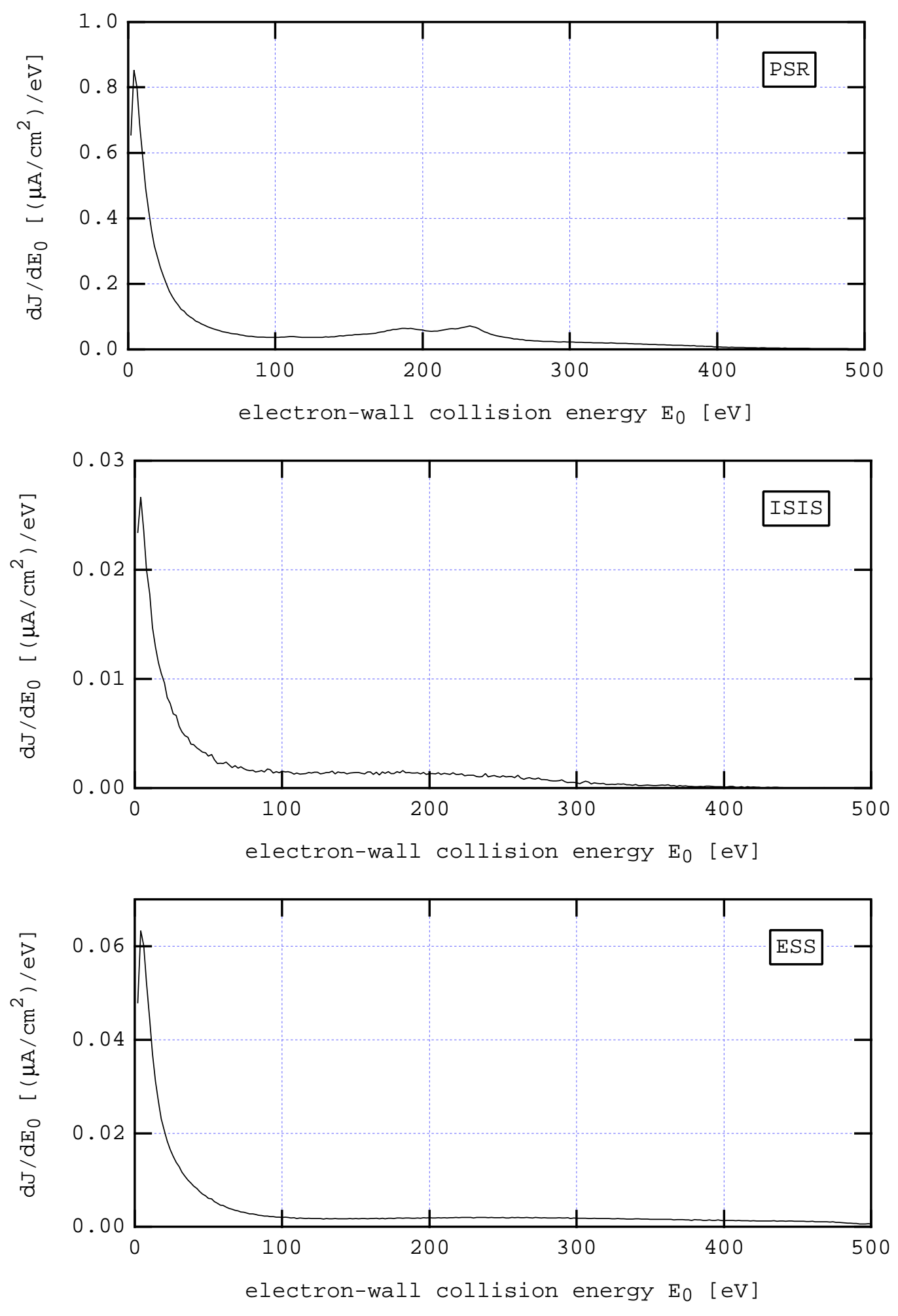

Figure 9: Time-averaged electron-wall collision energy spectrum. The normalization is such that the integral over $E_{0}$ gives the time-averaged electron-wall flux $J$. 

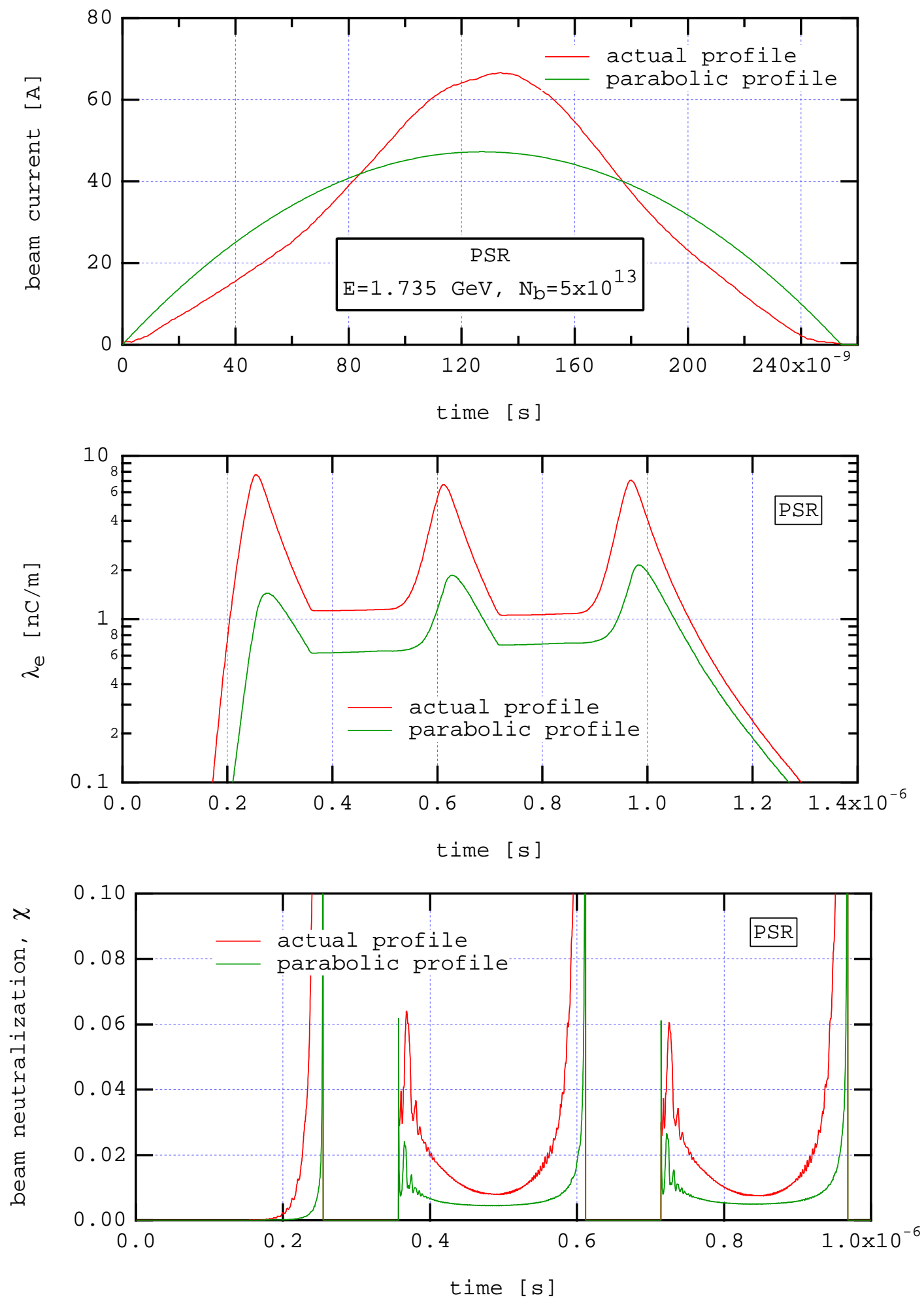

Figure 10: Bunch current, electron-cloud line density $\lambda_{e}$, and beam neutralization factor $\chi$ for the PSR assuming either the actual bunch profile or a parabolic profile. 

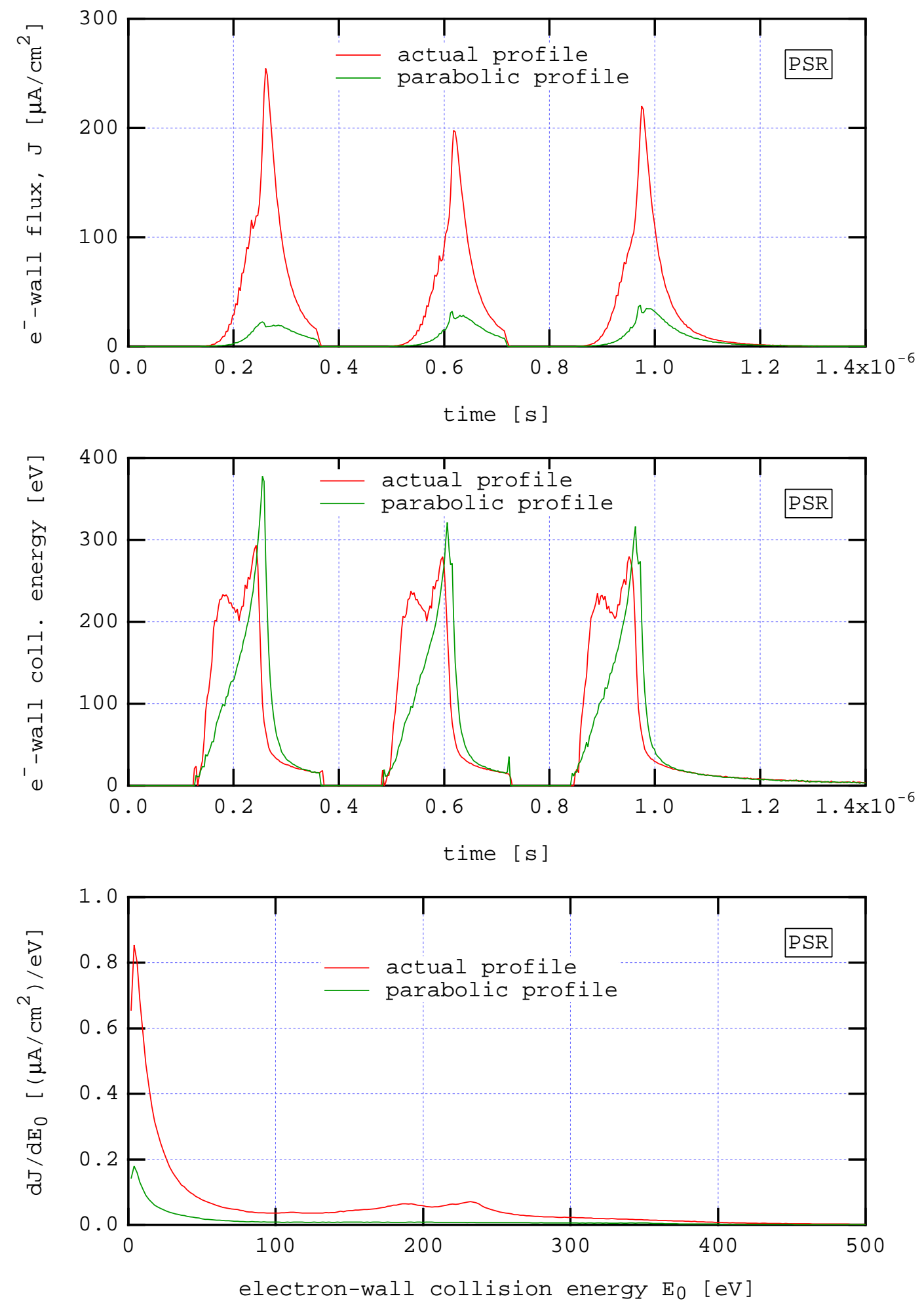

Figure 11: Electron-wall flux $J$, electron-wall collision energy, and time-averaged electron-wall energy spectrum for the PSR assuming either the actual bunch profile or a parabolic profile. 